\title{
Board 7: Partnership for Equity: Cultivating Inclusive Professional Identi- ties for Engineers and Computer Scientists across Four Unique Institutional Climates
}

\section{Dr. Rebecca A Atadero, Colorado State University}

Rebecca Atadero is an associate professor in the Department of Civil and Environmental Engineering at Colorado State University, specializing in structural engineering. She conducts research on the inspection, management and renewal of existing structures, and on diversity, equity and inclusion in engineering education.

\section{Dr. A.M. Aramati Casper, Colorado State University}

Dr. Aramati Casper is an education researcher and ecologist. She is currently a post doctoral fellow at Colorado State University doing research on diversity, inclusion, and social justice in undergraduate engineering classrooms.

\section{Dr. Karen E Rambo-Hernandez, West Virginia University}

Karen E. Rambo-Hernandez is an associate professor at West Virginia University in the College of Education and Human Services in the department of Learning Sciences and Human Development. In her research, she is interested the assessment of student learning, particularly the assessment of academic growth, and evaluating the impact of curricular change.

\section{Dr. Christina Paguyo, University of Denver}

Christina H. Paguyo, PhD, is the Director of Academic Assessment at the University of Denver. Her research interests focus on designing and examining educational environments grounded in research, theory, and equity.

\section{Dr. Jody Paul, Metropolitan State University of Denver}

Professor of Computer Science. Member of ABET Computing Accreditation Commission.

\section{Dr. Ronald R. DeLyser, University of Denver}

Ronald R. DeLyser is currently an Associate Professor of Electrical and Computer Engineering in the Department of Electrical and Computer Engineering the University of Denver where he has been on the faculty since 1986. He has received all of his degrees in Electrical Engineering: the B.S. degree from the University of Florida, Gainesville, in 1974; the M.S. degree from the University of New Mexico, in 1978; and the Ph.D. degree from the University of Colorado, Boulder in 1991. Dr. DeLyser, a member of the U.S. Air Force between 1965 and 1986, held a teaching position at the United States Air Force Academy, served as a development engineer at the Air Force Weapons Laboratory at Kirtland AFB in New Mexico and was the Requirements Officer for the Nellis AFB Ranges in Nevada. Prior to 2000, his research areas included pedagogy, outcomes based assessment, the study of periodic gratings used as antennas and in antenna systems, high power microwave interactions with large complex cavities, anechoic chambers, and anechoic chamber absorbing materials. Since 2000, he has been concentrating on engineering education pedagogy, engineering program accreditation, and outcomes based assessment for both engineering programs and general education, and inclusive excellence for engineering an computer science programs. 


\section{Partnership for Equity: Cultivating Inclusive Professional Identities for Engineers and Computer Scientists across Four Unique Institutional Climates}

The Partnership for Equity (P4E) project is funded under the NSF IUSE (Improving Undergraduate STEM Education) program. The goal of the project is to cultivate inclusive professional identities in engineering and computer science students. The project defines inclusive professional identities in terms of four key assets that students with inclusive professional identities will possess: (a) the necessary technical knowledge, skills, and abilities to work in their chosen field, (b) an appreciation for how all kinds of diversity strengthen engineering and computer science as disciplines, (c) knowledge of how to act in inclusive ways and create inclusive environments within their fields and (d) preparation to consider a diverse population of users when designing. Of note, the project espouses a broad definition of diversity [1], which includes diversity in terms of problem-solving approaches, personalities, and demographic characteristics as well as cognitive, social, and personal characteristics. While recognizing the need for a broad definition of diversity, project activities also place emphasis on understanding the experiences of traditionally marginalized and underrepresented populations in engineering and computing such as people of color, people with disabilities, and women.

Project activities are currently occurring at four partner campuses Colorado State University, Metropolitan State University of Denver, University of Denver and West Virginia University. The project builds on a prior pilot grant and is driven by three primary objectives: (1) to transfer first-year diversity and inclusion activities to three new campuses; (2) to develop, implement and assess diversity and inclusion activities for engineering and computing courses at the sophomore and junior level; and (3) to track students as they move through their degree programs to longitudinally assess the efficacy of the diversity and inclusion activities. CSU, DU and WVU are all predominately white institutions. MSU was recently designated as a Hispanic Serving Institution.

The poster accompanying this paper will highlight significant products from the second year of the five-year project with respect to four topics: activities to promote effective and inclusive teamwork, activities to teach students about diversity and equity in an engineering or computing context, efforts to engage and train faculty at each campus, and preliminary findings from project assessment.

Activities to promote effective and inclusive teamwork

A challenging, but essential part of this project is to identify and work with partnering engineering and computer science faculty. As the project expands, many of the faculty interested in participating teach existing courses with teamwork and have wanted to improve how teams function in their classes. The specific needs mentioned by faculty members are (a) encouraging teams to value and welcome diversity, and (b) working effectively in diverse teams. Several 
activities $(n=12)$ have been developed and are currently being piloted. For example, in one assignment, students were asked to identify a product that did not work well for the entire population (e.g., the pen-tether on credit card screens is sometimes too short for left-handed people) and consider how to construct teams that are more likely to consider a wide variety of users. A second assignment addressed the need for psychological safety [2] in teams via a case study of the NASA Columbia disaster. A third assignment had students watch TedX talks related to why diversity makes teams smarter and reflect on how the students should consider diversity in teams as a strength and a highly desirable quality. Existing activities and documents about team norms, team compacts and conflict resolution have also been updated and refined to set a more inclusive tone in these classrooms.

Activities to teach students about diversity within the engineering or computing context

This portion of the project has focused on developing activities that fit within technical engineering or computing courses (with or without teamwork) and relate directly to the course content. The poster will discuss at least two example assignments. The first assignment is for Engineering Mechanics: Dynamics and asks students to consider the impact of seatbelts and airbags in car crashes and the effectiveness of these safety devices for different populations of people, including drivers that are older or have larger bodies, children, and women. Students first completed a computation problem considering different occupant masses and sizes, then answered a series of reflective questions and read an article about research into different sized crash-test dummies.

The second assignment is a more course flexible assignment that uses a jigsaw approach to have students consider the perspectives of different stakeholders for an engineering design, product or process and to explore the privilege associated with different stakeholders. The assignment was first implemented for civil engineering students in a civil engineering materials course studying sustainability and the Envision sustainability rating system [3]. The assignment considered a large regional highway reconfiguration that has been in planning and design phases for nearly 20 years and recently broke ground. The project has severe impacts on a community of low socioeconomic status whose residents are predominantly people of color. Students read a variety of regional news sources and discussed the project in small groups and as a larger class. Initial responses were promising, but they indicated that as this type of assignment is refined and adapted for other engineering disciplines and computer science students, a more sustained interaction with the concept and implications of privilege and a variety of examples would be more impactful for students.

Efforts to engage and train faculty at each campus

The goals of this project cannot be achieved without increasing faculty understanding and appreciation for diversity, equity, and inclusion topics. This portion of the poster will describe opportunities and barriers to engaging faculty at all four project campuses. Each of the four campuses has unique opportunities and barriers related to the culture at each institution, the 
programs where implementation is occurring, and the students served by each institution. A new effort is planned at one of the partner institutions during spring and the poster will share up to date findings from this initiative.

\section{Preliminary findings from project assessment}

This section of the poster will highlight findings from across the project activities, including effectiveness of the first-year activities, and preliminary data describing the impact of teamwork and technical content based activities. At one of the expansion campuses, we rolled out the interventions in a smaller number of classes. Three instructors agreed to pilot a set of six activities, four homework assignments with follow up in-class discussions and two out-of-class experiences with reflection questions done via the university course management system. The instructors taught a total of eight classes, and each instructor had an equal number of courses that were taught "business-as-usual" and with the six intervention activities. Students were assessed four times over the semester using the Valuing Diversity and Enacting Inclusion in Engineering scale [4]. The four factors on the scale were engineers should value diversity to: (a) fulfill a greater purpose, $r=.90$, and (b) serve customers better, $r=.81$, and further engineers should (c) challenge discriminatory behavior, $r=.89$ and (d) promote a healthy team culture, $r=.85$.

Four models were run with mean scores on each of the four factors as the dependent variable and student participation in an intervention, time, and the interaction of time and student participation as the independent variables. Of the four factors, students in the two sections only differed on the final factor, promote a healthy culture $\left(R^{2}=.01, p=.024\right)$. The model implied results are illustrated in Figure 1.

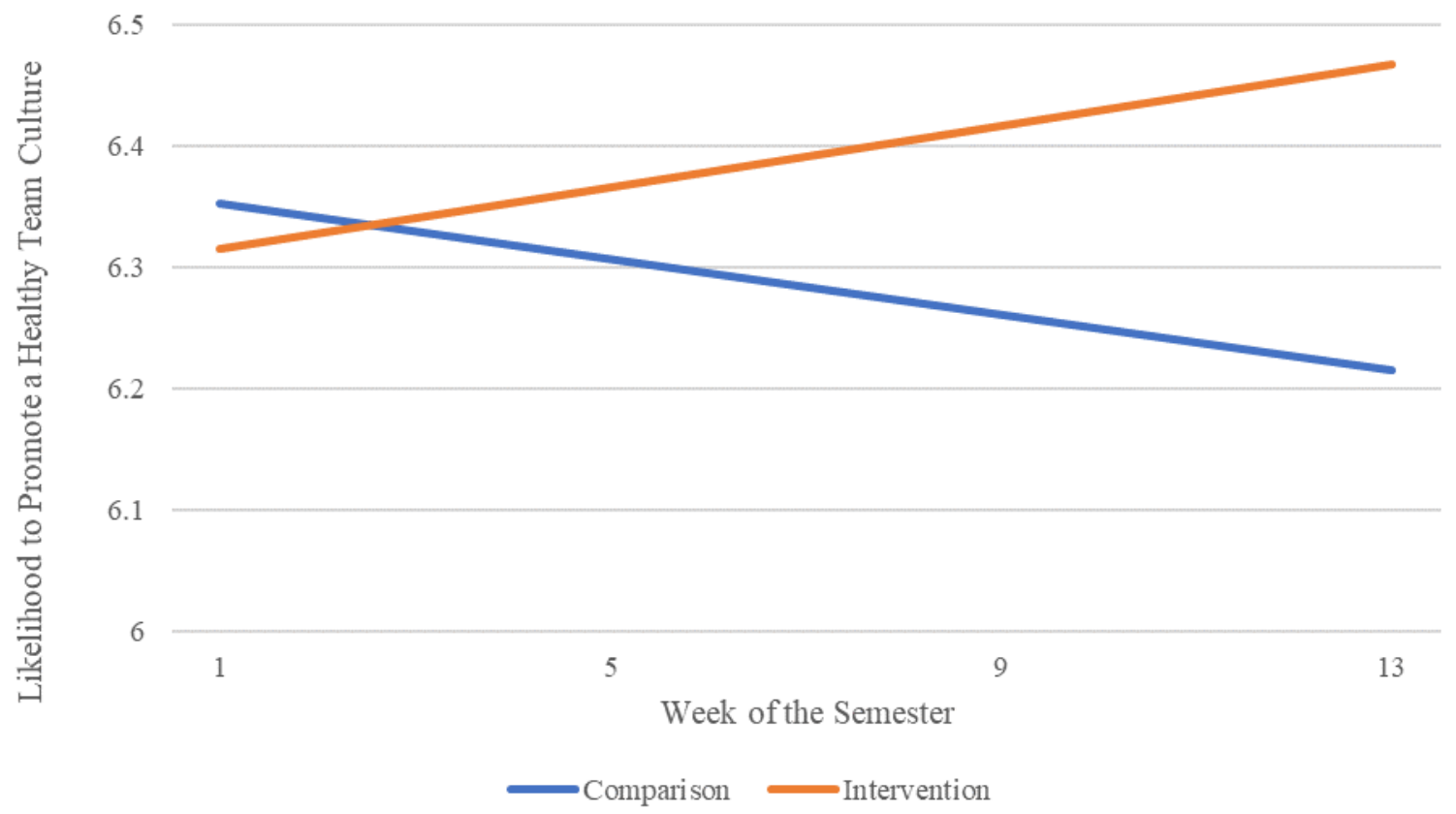

Figure 1. Model results comparing likelihood to promote a healthy culture between sections with 
and without intervention activities. Reported differences are small, but promising, particularly after only one semester.

Conclusion

Changes are needed in the culture of engineering and computer science programs to make students of all identities feel welcome, valued, and affirmed. The project is working to promote culture changes within classrooms at four unique campuses. Diversity, equity and inclusion work is difficult, yet rewarding when successes are achieved. Members of the project team are happy to share lessons learned with change agents on other campuses.

\section{Acknowledgements}

This material is based upon work supported by the National Science Foundation under Grant Nos. 1726268, 1725880 and 1726088 . Any opinions, findings, and conclusions or recommendations expressed in this material are those of the author(s) and do not necessarily reflect the views of the National Science Foundation.

\section{References}

[1] S. Page, The Difference. Princeton, NJ: Princeton University Press, 2008.

[2] Edmondson, A. (1999). Psychological safety and learning behavior in work teams. Administrative science quarterly, 44(2), 350-383.

[3] Institute for Sustainable Infrastructure. https://sustainableinfrastructure.org/

[4] Rambo-Hernandez, K. E., Atadero, R. A., Paguyo, C., \& Schwartz, J. “Inclusive Engineering Identities: Two New Surveys to Assess Students’ Inclusive Values and Behaviors.” ASEE Annual Conference. Columbus, OH, USA. June 25-28, 2017. 